\title{
Enhancement of astaxanthin production in Xanthophyllomyces dendrorhous by efficient method for the complete deletion of genes
}

Keisuke Yamamoto ${ }^{1 \dagger}$, Kiyotaka Y. Hara ${ }^{2,3 \dagger}{ }^{\dagger}$, Toshihiko Morita ${ }^{1}$, Akira Nishimura ${ }^{4}$, Daisuke Sasaki ${ }^{5}$, Jun Ishii ${ }^{5}$, Chiaki Ogino ${ }^{1}$, Noriyuki Kizaki ${ }^{6}$ and Akihiko Kondo ${ }^{1,5^{*}}$

\begin{abstract}
Background: Red yeast, Xanthophyllomyces dendrorhous is the only yeast known to produce astaxanthin, an antioxidant isoprenoid (carotenoid) widely used in the aquaculture, food, pharmaceutical and cosmetic industries. The potential of this microorganism as a platform cell factory for isoprenoid production has been recognized because of high flux through its native terpene pathway. Recently, we developed a multiple gene expression system in $X$. dendrorhous and enhanced the mevalonate synthetic pathway to increase astaxanthin production. In contrast, the mevalonate synthetic pathway is suppressed by ergosterol through feedback inhibition. Therefore, releasing the mevalonate synthetic pathway from this inhibition through the deletion of genes involved in ergosterol synthesis is a promising strategy to improve isoprenoid production. An efficient method for deleting diploid genes in X. dendrorhous, however, has not yet been developed.
\end{abstract}

Results: Xanthophyllomyces dendrorhous was cultivated under gradually increasing concentrations of antibiotics following the introduction of antibiotic resistant genes to be replaced with target genes. Using this method, double CYP61 genes encoding C-22 sterol desaturases relating to ergosterol biosynthesis were deleted sequentially. This double CYP61 deleted strain showed decreased ergosterol biosynthesis compared with the parental strain and single CYP61 disrupted strain. Additionally, this double deletion of CYP61 genes showed increased astaxanthin production compared with the parental strain and the single CYP61 knockout strain. Finally, astaxanthin production was enhanced by 1.4-fold compared with the parental strain, although astaxanthin production was not affected in the single CYP61 knockout strain.

Conclusions: In this study, we developed a system to completely delete target diploid genes in X. dendrorhous. Using this method, we deleted diploid CYP61 genes involved in the synthesis of ergosterol that inhibits the pathway for mevalonate, which is a common substrate for isoprenoid biosynthesis. The resulting decrease in ergosterol biosynthesis increased astaxanthin production. The efficient method for deleting diploid genes developed in this study has the potential to improve industrial production of various isoprenoids in $X$. dendrorhous.

Keywords: Gene deletion, Astaxanthin production, Yeast, Xanthophyllomyces dendrorhous, Metabolic engineering

\footnotetext{
*Correspondence: akondo@kobe-u.ac.jp

${ }^{\dagger}$ Keisuke Yamamoto and Kiyotaka Y. Hara contributed equally to this work

${ }^{5}$ Graduate School of Science, Technology, and Innovation, Kobe

University, 1-1 Rokkodai-cho, Nada-ku, Kobe, Hyogo 657-8501, Japan

Full list of author information is available at the end of the article
} 


\section{Background}

Carotenoids are widely distributed in nature and are exclusively synthesized by plants and microorganisms [1]. Carotenoids belong to the natural compounds class of terpenes (isoprenoids) [2]. Bioproduction of pharmaceutically important carotenoids such as artemisinin and Taxol precursor, has been accomplished by genetically engineered, well-characterized microorganisms such as Saccharomyces cerevisiae and Escherichia coli [3, 4]. Recently, however, Melillo, et al. [5] showed the potential of red yeast, Phaffia rhodozyma (sexual form, Xanthophyllomyces dendrorhous), as a platform microorganism for isoprenoid production because of the higher rates of flux through its native terpene pathway compared with S. cerevisiae and E. coli. X. dendrorhous has been studied as a promising candidate microorganism for maximizing production of the carotenoid astaxanthin (3, $3^{\prime}$-dihydroxy- $\beta$, $\beta$-carotene- 4 , 4'-dione; $\left.\mathrm{C}_{40} \mathrm{H}_{52} \mathrm{O}_{4}\right)$. Astaxanthin is used in fine chemical industries such as the food, pharmaceutical, and cosmetics industries because of its antioxidant properties [6,7].

We previously identified strong promoters for the expression of target genes and developed a multi-gene expression system in $X$. dendrorhous [7, 8]. Astaxanthin production was enhanced by metabolic engineering using this system [8]. In contrast, high astaxanthin-producing mutant strains were previously obtained from randomly mutated strains $[9,10]$. In most of these strains, the critical mutations for enhancing astaxanthin biosynthesis have not been identified; however, these most likely involve a lack of genes affecting astaxanthin biosynthesis. Thus, gene disruption was a very promising strategy to enhance astaxanthin production. However, a problem with random mutagenesis is the potential occurrence of unexpected mutations that could have negative effects on industrial astaxanthin production. Indeed, though astaxanthin content was increased by random mutagenesis, cell growth was inhibited [10]. A decrease in the final cell concentration has a negative effect on the volumetric concentration of astaxanthin, the most important factor for industrial astaxanthin production. Thus, a targeted gene disruption technique is required for deleting genes that negatively affect astaxanthin production. A gene deletion technique is essential for further improvement of astaxanthin production in $X$. dendrorhous. Two methods for the deletion of target genes in $X$. dendrorhous have been reported previously. Mauricio et al. [11] reported the double recombinant method in 2008, where two different concentrations of antibiotics were used. Some researchers, including the authors, tried and failed to delete target genes using the double recombinant method [12, 13]. In contrast, Iris et al. [14] reported a method to delete target diploid genes using two different antibiotic resistant genes. This method can be used for deletion of only two diploid genes because only four antibiotic-resistant genes have been known to work as antibiotic selection markers in X. dendrorhous.

In this study, we developed an easy and novel method for the double deletion of diploid target genes in X. dendrorhous using one antibiotic resistant gene that saves selection markers. The outline of this new method is shown in Fig. 1. Using this method, we deleted both diploid CYP61 genes encoding C-22 sterol desaturases, which negatively regulate hydroxymethylglutaryl-coenzyme A (HMG-CoA) synthase (HmgS) and HMG-CoA reductase $(\mathrm{HmgR})$ in the ergosterol biosynthesis, the rate-limiting enzymes in the mevalonate synthetic pathway [14] (Fig. 2). The double deletion of diploid CYP61 genes decreased the ergosterol biosynthesis, leading to improved astaxanthin production.

\section{Results and discussion}

\section{An efficient method for double deletion of target genes in X. dendrorhous}

We developed an efficient method for double deletion of target diploid genes in $X$. dendrorhous through a single genetic transformation event followed by cultivation with gradually increasing concentrations of antibiotics. This study targeted the diploid CYP61 genes for deletion to develop the method. It was expected that double deletion of CYP61 genes would suppress synthesis of ergosterol and, therefore, increase astaxanthin content by releasing the mevalonate biosynthesis pathway from feedback inhibition by ergosterol.

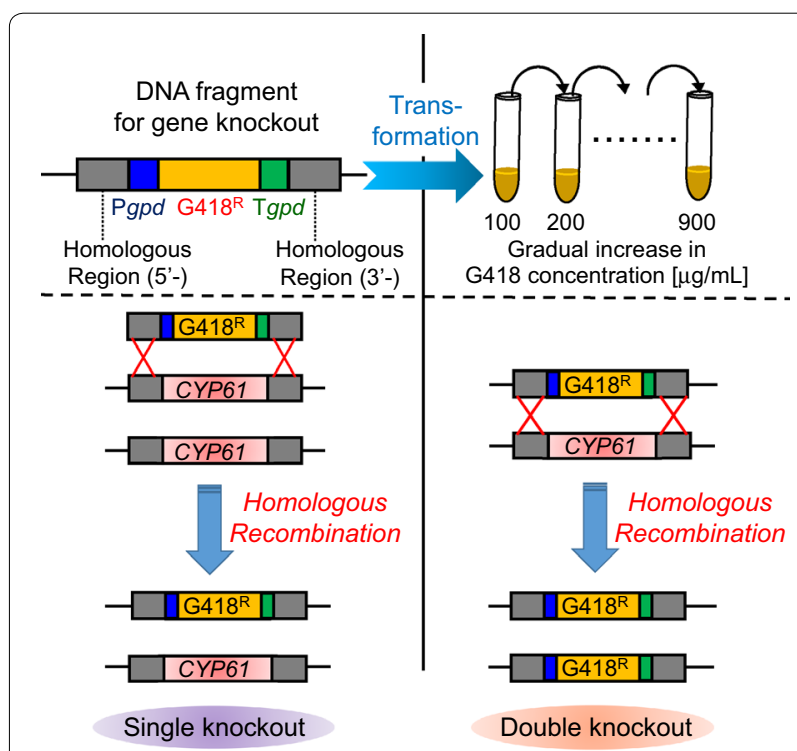

Fig. 1 The outline of a novel method for double deletion of diploid target genes in $X$. dendrorhous using one drug-resistant gene 


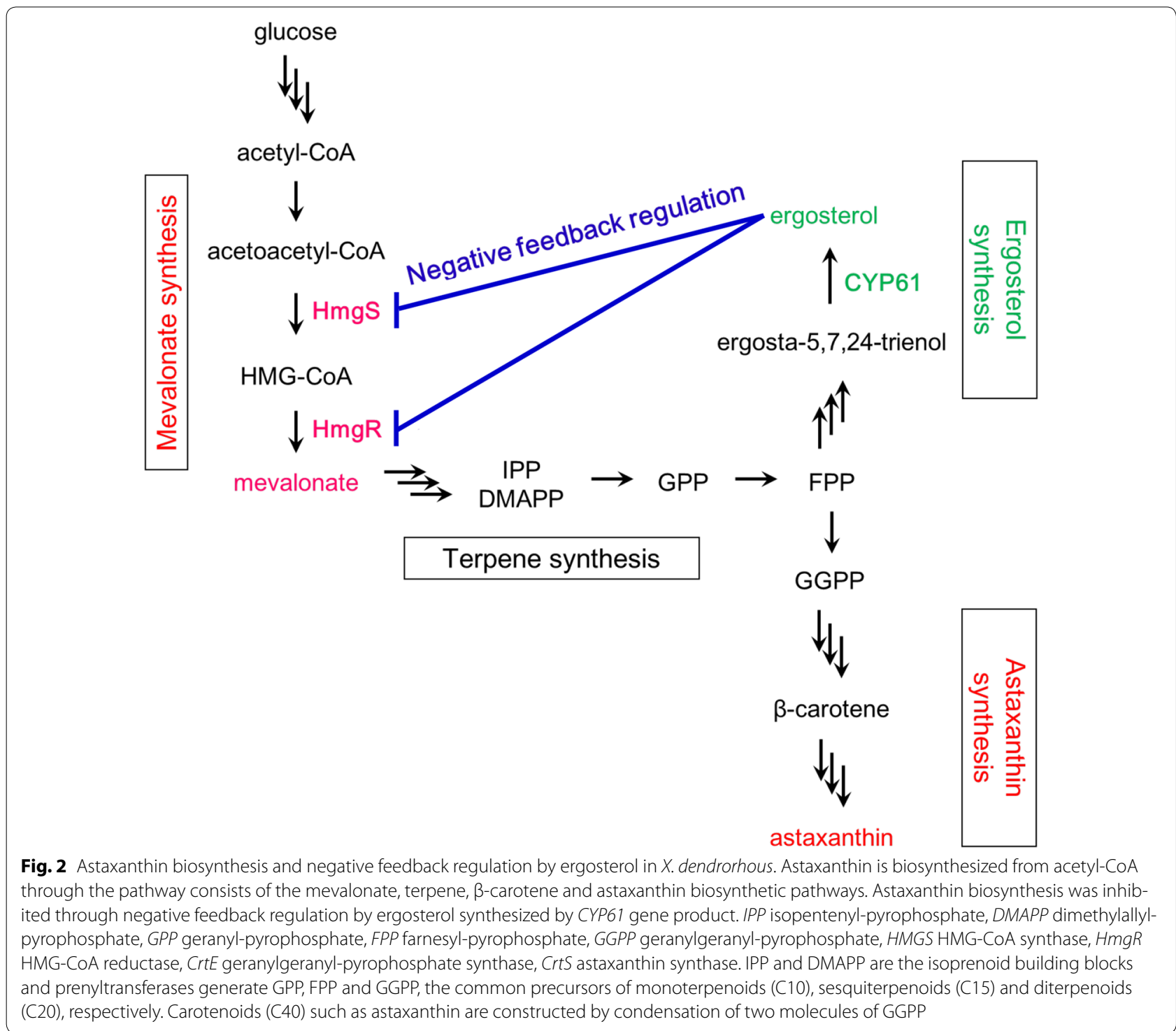

Firstly, the plasmid pKF-G418-gCYP61 digested with NdeI was transformed into the parental $X$. dendrorhous host strain to construct the CYP61 single disruption strain $[\triangle C Y P 61(+,-)]$. The genotype of the $\triangle C Y P 61(+,-)$ strain was confirmed by PCR using specific primers for the CYP61 gene and G418 resistant cassette (Fig. 3a, b). Specific fragments of CYP61 (A and $B)$ were confirmed in both the parental host strain and $\triangle C Y P 61(+,-)$ strain. Conversely, the specific fragments for the G418 resistant cassette (C and D) were confirmed in the $\triangle C Y P 61(+,-)$ strain, but not in the parental host strain. Furthermore, the theoretical length of fragment $\mathrm{E}$ from the G418 resistant cassette (4.5 kbp) appeared in the $\triangle C Y P 61(+,-)$ strain, while the CYP61 gene fragment $(7.5 \mathrm{kbp})$ appeared in both the parental host strain and the $\triangle C Y P 61(+,-)$ strain. These results indicated that a single CYP61 gene was disrupted in the $\triangle C Y P 61(+,-)$ strain. This first disruption of a single target gene would be replaced using a genome editing technology such as the CRISPR-Cas9 system.

Next, we carried out disruption of both CYP61 genes because ergosterol biosynthesis of $\triangle C Y P 61(+,-)$ strain was almost equal to that of the parental strain as shown in Fig. 4 and described in detail in the following section "Ergosterol biosynthesis". The sequential operations were carried out to cultivate $\triangle C Y P 61(+,-)$ strain up to a total

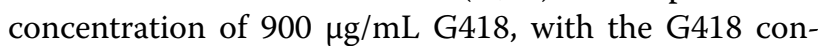
centration increasing in $100 \mu \mathrm{g} / \mathrm{mL}$ intervals. Dilutions of the culture with $900 \mu \mathrm{g} / \mathrm{mL}$ G418 were plated on solid YM medium containing $900 \mu \mathrm{g} / \mathrm{mL}$ G418 to obtain single 


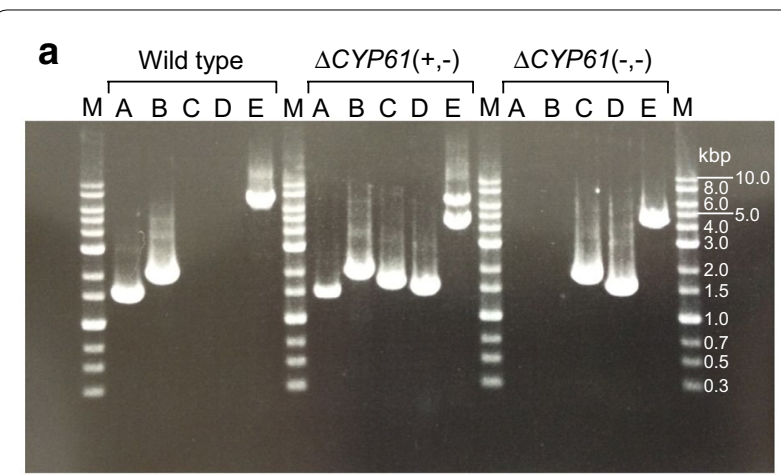

b
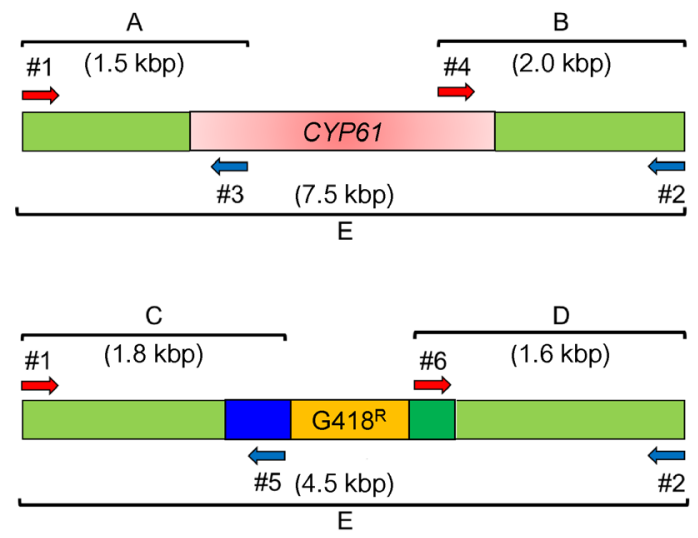

Fig. 3 PCR-based analysis of CYP61 mutants. a The diagram shows the amplification target and each set of primers. $\mathbf{b}$ The gel shows PCR reactions performed with different sets of primers and genomic DNA from the parental strain (WT), $\triangle \operatorname{CYP} 61(+,-)$ strain and $\triangle \operatorname{CYP61}(-,-)$ strain. Lanes $A, B, C, D$ and $E$ represent the fragment between primers \#1 and \#3 (1.5 kbp), \#2 and \#4 (2.0 kbp), \#1 and \#5 (1.8 kbp), \#2 and \#6 (1.6 kbp), and \#1 and \#2 (7.5 kbp for CYP61 gene and $4.5 \mathrm{kbp}$ for $\mathrm{G} 418^{\mathrm{R}}$ gene), respectively. The estimated sizes of the PCR fragments were represented in parenthesis. Lane $M$ represent DNA marker and numbers on the right side show molecular weights of DNA marker

colonies, some of which were then selected. The genotype of the $\triangle C Y P 61(-,-)$ strain was confirmed by PCR using specific primers for the CYP61 gene and the G418 resistant cassette (Fig. 3). Like the $\triangle C Y P 61(+,-)$ strain, the specific fragments of the G418 resistant cassette (C and D) and the theoretical length of fragment $E$ from the G418 resistant cassette ( $4500 \mathrm{bp}$ ) were confirmed in the $\triangle C Y P 61(-,-)$ strain. In contrast, the specific fragments for CYP61 (A and B) completely disappeared in the $\triangle C Y P 61(-,-)$ strain. These results indicated that both diploid CYP61 genes were successfully deleted in the $\triangle C Y P 61(-,-)$ strain. Five $\triangle C Y P 61(-,-)$ strains were obtained from 60 colonies. Homologous recombination between the disrupted and non-disrupted allele regions was used to delete the CYP61 gene from the $\triangle C Y P 61(+$,
-) strain. This was achieved by selection pressure applied through a gradual increase in antibiotic concentration.

\section{Ergosterol biosynthesis}

Ergosterol biosynthesis in the parental host strain, $\triangle C Y P 61(+,-)$ and $\triangle C Y P 61(-,-)$ was evaluated by reversed phase high-performance liquid chromatography (RP-HPLC). Chromatograms of ergosterol extractions from the parental host strain, $\triangle C Y P 61(+,-)$ strain and $\triangle C Y P 61(-,-)$ strain are shown in Fig. 4a. In the parental host strain and $\triangle C Y P 61(+,-)$ strain, a peak at $280 \mathrm{~nm}$ at $15.6 \mathrm{~min}$ was observed and identified as the ergosterol peak, confirmed by the addition of standard ergosterol. Conversely, this ergosterol peak was not observed in the $\triangle C Y P 61(-,-)$ strain. As shown in the time course of ergosterol concentrations (Fig. 4b), ergosterol biosynthesis in the parental host strain and $\triangle C Y P 61(+,-)$ strain almost stopped after $24 \mathrm{~h}$ of cultivation. In contrast, ergosterol biosynthesis was not observed in the $\triangle C Y P 61(-,-)$ strain for more than $72 \mathrm{~h}$ from the start of the cultivation.

\section{Astaxanthin fermentation}

Astaxanthin production in the parental host strain, $\triangle C Y P 61(+,-)$ and $\triangle C Y P 61(-,-)$ was evaluated by RPHPLC. Cell growth, intracellular astaxanthin content, and volumetric astaxanthin concentrations extracted from these strains are shown in Fig. 5. After $24 \mathrm{~h}$ of fermentation, cell growth rate had been obviously slower than that before $24 \mathrm{~h}$ of fermentation, even in the parental host strain (Fig. 5a), which would explain why additional ergosterol biosynthesis was not observed after $24 \mathrm{~h}$ (Fig. 4b). The cell concentration of the $\triangle C Y P 61(-,-)$ strain was lower than that of the parental host strain until after $48 \mathrm{~h}$ of fermentation while that of the $\triangle C Y P 61(+,-)$ strain was almost the same as the parental strain (Fig. 5a). Because ergosterol is a component of the cell membrane, this slower cell growth is caused by the suppression of ergosterol biosynthesis. In contrast, after $48 \mathrm{~h}$ of fermentation, the cell growth of all strains had stopped because glucose would be depleted.

Conversely, astaxanthin production decreased after $24 \mathrm{~h}$ of fermentation and was almost stopped after $48 \mathrm{~h}$ in the parental host strain and $\triangle C Y P 61(+,-)$ strain. In the $\triangle C Y P 61(-,-)$ strain, astaxanthin production instead continuously increased after $24 \mathrm{~h}$ of fermentation and gradually increased even after $48 \mathrm{~h}$ of fermentation (Fig. 5b). Finally, the volumetric astaxanthin concentration after $72 \mathrm{~h}$ of fermentation of the $\triangle C Y P 61(-,-)$ strain $(1.65 \mathrm{mg} / \mathrm{L})$ was approximately 1.4 -fold higher than that of the parental host strain $(1.22 \mathrm{mg} / \mathrm{L})$, although that of $\triangle C Y P 61(-,-)$ strain $(1.25 \mathrm{mg} / \mathrm{L})$ was not changed (Fig. $5 \mathrm{c}$ ). These results indicate that astaxanthin production was suppressed through feedback inhibition of the mevalonate pathway by ergosterol. The double deletion of 

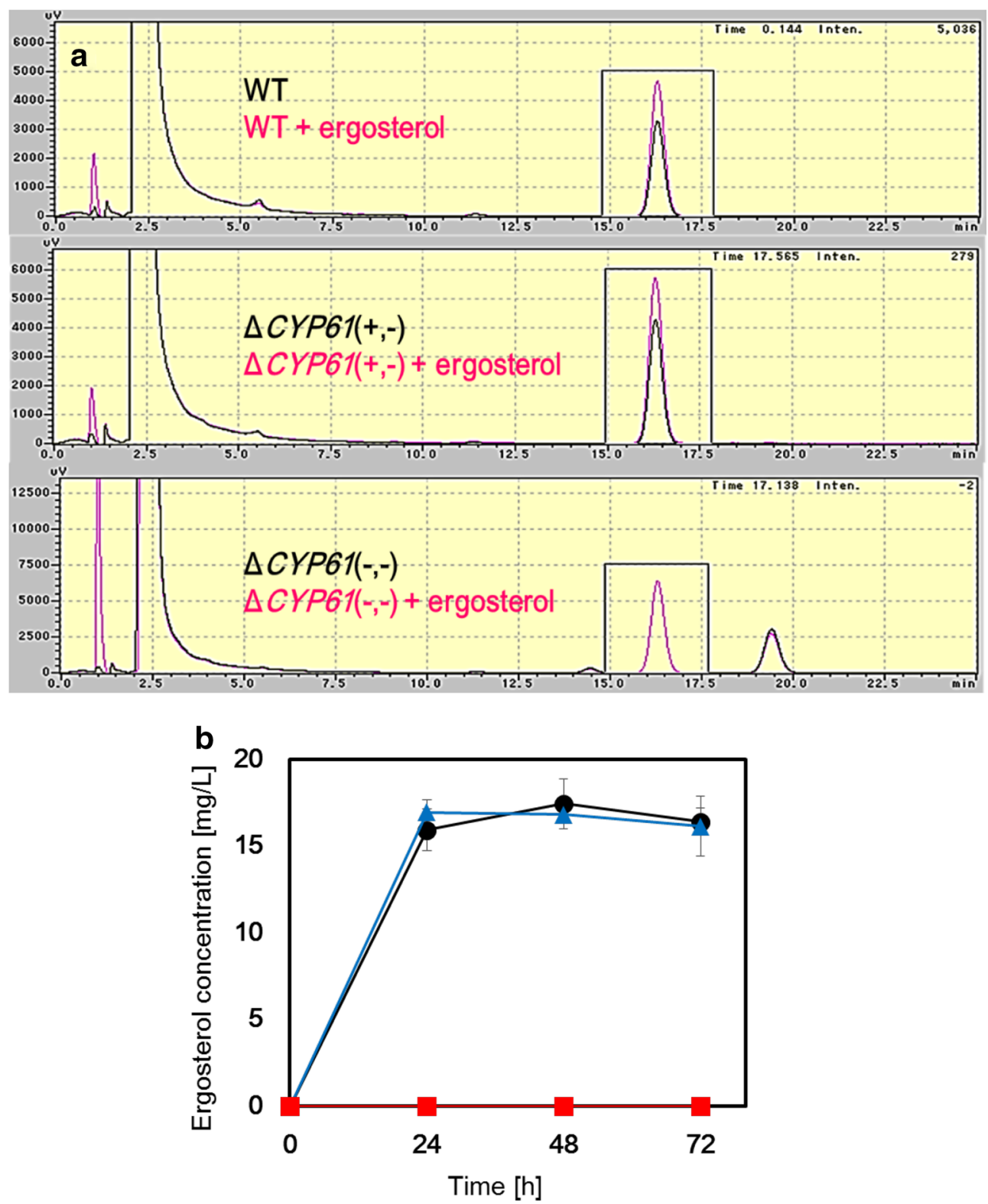

Fig. 4 Ergosterol biosynthesis. a RP-HPLC ergosterol analysis from the parental host strain, $\triangle C Y P 61(+,-)$ and $\triangle C Y P 61(-,-)$. Chromatograms (at $280 \mathrm{~nm}$ ) correspond to ergosterol extracted from strains as described in the "Methods" section. Ergosterol extracts (black) from the parental host strain, $\triangle C Y P 61(+,-)$ strain and $\triangle C Y P 61(-,-)$ strain were analyzed with co-injection of ergosterol standard (pink). b Ergosterol production in the parental host strain, $\triangle C Y P 61(+,-)$ strain and $\triangle C Y P 61(-,-)$ strain. Circle (black), triangle (blue) and square (red) symbols represent values of the parental host strain, $\triangle \operatorname{CYP61}(+,-)$ strain and $\triangle \operatorname{CYP61}(-,-)$ strain, respectively. The values are means and the error bars show the standard deviation $(n=3)$

diploid CYP61 genes was critical to release this feedback inhibition, with the single disruption of CYP61 having almost no effect on inhibition. The physiological concentration of ergosterol needed for feedback inhibition of the mevalonate pathway can be estimated to be 10 -fold higher than the concentration of astaxanthin according to the results of Figs. $4 \mathrm{~b}$ and $5 \mathrm{c}$. The fold-change increase in astaxanthin production following CYP61 gene deletion (1.6-fold) was almost equal to that (1.5-fold) caused by overexpression of genes involved in astaxanthin biosynthetic metabolism [8]. In contrast to these overexpression strategies that used wild-type strains as the host strain, a greater fold-change increase in astaxanthin production (3.4-fold) was observed when an astaxanthin-hyper 

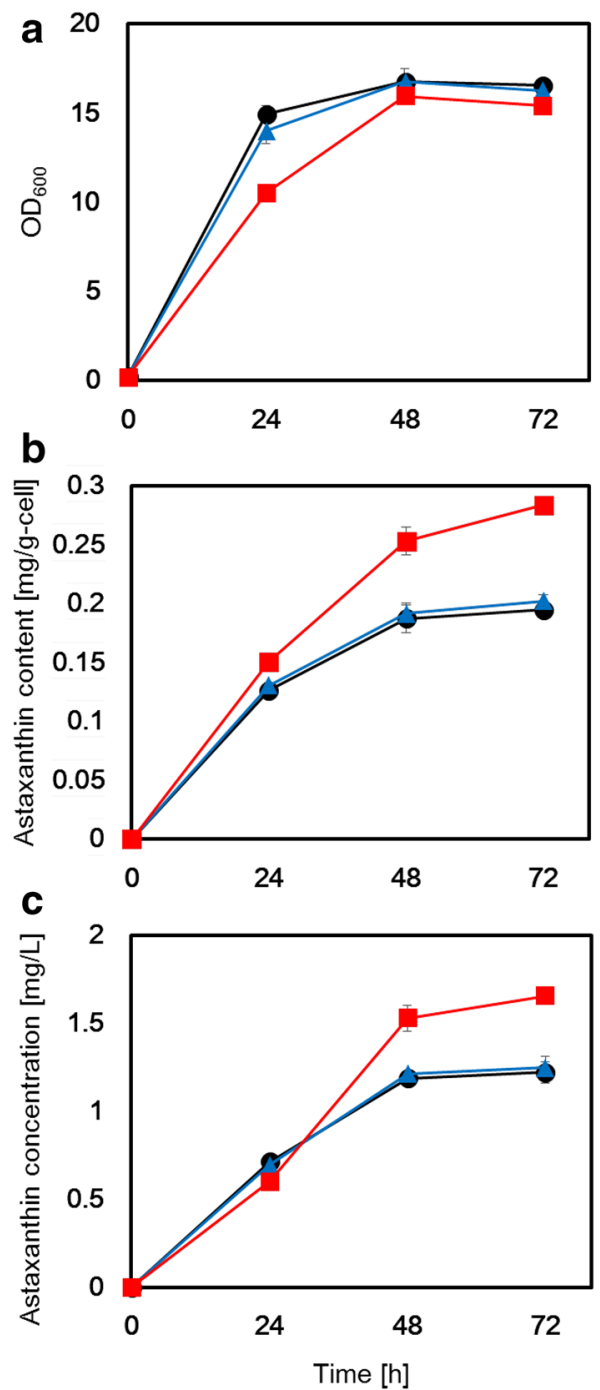

Fig. 5 RP-HPLC ergosterol analysis from the parental host strain, $\triangle C Y P 61(+,-)$ strain and $\triangle C Y P 61(-,-)$ strain respectively, and astaxanthin production in each. a Cell concentration $\left(\mathrm{OD}_{600}\right)$; b intracellular astaxanthin content (mg/g-cell). c volumetric astaxanthin concentration (mg/L). Circle (black), triangle (blue) and square (red) symbols represent values of the parental host strain, $\triangle \operatorname{CYP} 61(+,-)$ strain and $\triangle C Y P 61(-,-)$ strain, respectively

producing mutant strain generated by random mutagenesis was used as a host strain [15]. However, the problem of random mutagenesis is reduced in the cell growth of the host strain compared with the wild-type strain [15] because unexpected mutations occurring through random mutagenesis, in addition to the expected mutations, result in enhanced astaxanthin biosynthesis $[9,10]$. In contrast to random mutagenesis, the disruption of target genes carried out using the gene disruption method developed in this study did not decrease the final cell concentration because of the absence of any unexpected mutations. The developed strain has the potential to be one of the best strains for further improvement of astaxanthin production through metabolic engineering because it has an original metabolic background.

\section{Conclusion}

The two previously described methods for deletion of target diploid genes in $X$. dendrorhous had significant room for improvement. In this study, we developed an easy, novel method for double deletion of target genes from the diploid genome of $X$. dendrorhous through a single genetic transformation event followed by cultivation with gradually increasing antibiotic concentrations. Compared with previous methods, this method allows for efficient deletion of target genes while saving antibiotic resistant markers for use in deleting or overexpressing additional target genes. This method makes it possible to obtain strains that have completely lost the target allele. Indeed, we deleted double diploid CYP61 genes using this method and achieved improvement of astaxanthin production in $X$. dendrorhous. This method has the potential to improve industrial production of various isoprenoids in $X$. dendrorhous without a decrease in cell growth.

\section{Methods}

\section{Strains and media}

NovaBlue (Novagen, Madison, WI, USA) was used as the $E$. coli host strain for recombinant DNA manipulation. $X$. dendrorhous (NBRC 10129) was used as the parental host strain for gene expression. E. coli transformants were grown in Luria broth medium $(10 \mathrm{~g} / \mathrm{L}$ tryptone, $5 \mathrm{~g} / \mathrm{L}$ yeast extract, and $5 \mathrm{~g} / \mathrm{L}$ sodium chloride) supplemented with $100 \mu \mathrm{g} / \mathrm{mL}$ ampicillin. Transformants of $X$. dendrorhous were cultured in YM medium (5 g/L tryptone, $3 \mathrm{~g} / \mathrm{L}$ yeast extract, $3 \mathrm{~g} / \mathrm{L}$ malt extract and $10 \mathrm{~g} / \mathrm{L}$ glucose). Yeast extract and malt extract were purchased from Becton-Dickinson (Sparks, MD, USA). Other chemicals were obtained from Nacalai Tesque (Kyoto, Japan) or Wako Chemicals (Osaka, Japan).

\section{Plasmid construction}

Target genes were cloned by PCR using KOD-Plus-Neo DNA polymerase (Toyobo, Osaka, Japan). Nucleotide sequences of the cloning primers for target genes are shown in Additional file 1. To construct the G418 resistant CYP61 disruption plasmid pKF-G418-gCYP61, the G418 resistance cassette was amplified by PCR from pKFG418-AAT [8] using the Pgpd-fw/Tgpd-rv forward and reverse primer set. The $5^{\prime}$ - and $3^{\prime}$-homologous regions of CYP61 were also amplified by PCR from $X$. dendrorhous genomic DNA. The forward primer and reverse primer sets used for these amplifications were g-CYP61-5'$\mathrm{fw} / \mathrm{g}-C Y P 61-5^{\prime}-\mathrm{rv}$ and g-CYP61-3'-fw/g-CYP61-3'-rv, 
respectively. These fragments were connected by PCR using g-CYP61-5'-fw/g-CYP61-3'-rv, generating the CYP61 disruption cassette. The length of the homologous sequence in the CYP61 disruption cassette was designed to be 1000 base pairs. This cassette was cloned into the NdeI digested pKF-G418-AAT fragment, which included $\mathrm{Amp}^{\mathrm{R}}$, using the In-Fusion ${ }^{\circledR}$ HD Cloning Kit (Takara, Shiga, Japan), to construct pKF-G418-gCYP61.

\section{Yeast transformation}

Transformation was carried out using the method described in previous reports $[11,16]$ with some modifications for the construction of competent cells as follows. $X$. dendrorhous parental strains were grown in $5 \mathrm{~mL}$ liquid YM medium at $22{ }^{\circ} \mathrm{C}$ with agitation at $250 \mathrm{rpm}$ for $24 \mathrm{~h}$. Sufficient volumes of each culture were inoculated into $150 \mathrm{~mL}$ liquid YM medium to achieve initial values of 0.03 optical density at $600 \mathrm{~nm}\left(\mathrm{OD}_{600}\right)$. Cultures were then grown at $22{ }^{\circ} \mathrm{C}$ with agitation at $120 \mathrm{rpm}$ for 16.5 h. The NdeI digested plasmids pKF-G418-gCYP61 were transformed into the parental $X$. dendrorhous host strain to construct the CYP61 single disruption strain $(\triangle C Y P 61(+,-))$.

\section{Double deletion of CYP61 genes from genome in $X$. dendrorhous}

The $\triangle C Y P 61(+,-)$ strain was cultivated in a test tube containing $5 \mathrm{~mL}$ of yeast mold (YM) media with $40 \mu \mathrm{g} /$ $\mathrm{mL}$ of G418, incubated at $22{ }^{\circ} \mathrm{C}$ with agitation at $250 \mathrm{rpm}$ for $24 \mathrm{~h}$. A $200 \mu \mathrm{L}$ aliquot of the culture was then inoculated into $5 \mathrm{~mL}$ YM medium plus $100 \mu \mathrm{g} / \mathrm{mL}$ of G418. After sufficient growth at $22^{\circ} \mathrm{C}$ with agitation at $250 \mathrm{rpm}$, a $200 \mu \mathrm{L}$ aliquot of the culture was inoculated into $5 \mathrm{~mL}$ YM medium plus $200 \mu \mathrm{g} / \mathrm{mL}$ G418. The sequential operations were carried out up to a total concentration of $900 \mu \mathrm{g} / \mathrm{mL}$ G418, with the G418 concentration increasing in $100 \mu \mathrm{g} / \mathrm{mL}$ intervals. Dilutions of the culture with $900 \mu \mathrm{g} / \mathrm{mL}$ G418 were plated on solid YM medium containing $900 \mu \mathrm{g} / \mathrm{mL}$ G418 to obtain single colonies, some of which were then selected. The genotype of the $\triangle C Y P 61(-,-)$ strain was confirmed by PCR using specific primers for the CYP61 gene and the G418 resistant cassette.

\section{Astaxanthin fermentation of $X$. dendrorhous strains}

Xanthophyllomyces dendrorhous strains with deleted target genes were grown in $5 \mathrm{~mL}$ liquid YM medium, also containing $40 \mu \mathrm{g} / \mathrm{mL}$ G418 as required, in test tubes at $22{ }^{\circ} \mathrm{C}$ with agitation at $250 \mathrm{rpm}$ for $24 \mathrm{~h}$. Sufficient volumes of each culture were inoculated into $80 \mathrm{~mL}$ liquid YM medium in a Sakaguchi flask to achieve initial $\mathrm{OD}_{600}$ values of 0.15 . Cells were then grown at $22^{\circ} \mathrm{C}$ with agitation at $120 \mathrm{rpm}$ for less than $72 \mathrm{~h}$.

\section{Extraction and RP-HPLC measurement of astaxanthin and ergosterol}

Cell concentration was measured as $\mathrm{OD}_{600}$ after culturing for the appropriate time. The cell mass (dry cell weight) was calculated by $0.3786 \times \mathrm{OD}_{600}$. To measure the intracellular astaxanthin content of $X$. dendrorhous mutants, harvested cells were suspended in $1 \mathrm{~mL}$ acetone. The cells were broken using a bead shaker (Shake Master NEO, BMS, Tokyo, Japan) with zirconia beads. The cell extract was centrifuged at $8000 \times g$ at $4{ }^{\circ} \mathrm{C}$ for $10 \mathrm{~min}$ and the supernatant was then diluted with an appropriate volume of acetone for cover the range of following HPLC assay.

Astaxanthin concentration was determined using a HPLC machine (Shimadzu, Kyoto, Japan) equipped with a Develosil ODS-HG-5 column (Nomura Chemical, Aichi, Japan). The operating conditions were a temperature of $25^{\circ} \mathrm{C}$, with acetonitrile/methanol/2-propanol $(85 / 10 / 5[\mathrm{v} / \mathrm{v}])$ as the mobile phase at a flow rate of $0.8 \mathrm{~mL} / \mathrm{min}$, and the detection was performed at $471 \mathrm{~nm}$ with a UV detector SPD-20A (Shimadzu).

Ergosterol concentration was also determined using a HPLC machine (Shimadzu) equipped with a Develosil ODS-HG-5 column (Nomura Chemical). The operating conditions were a temperate of $30{ }^{\circ} \mathrm{C}$, with methanol/ water $(97 / 3[\mathrm{v} / \mathrm{v}])$ as the mobile phase at a flow rate of $1.0 \mathrm{~mL} / \mathrm{min}$, and the detection was performed at $280 \mathrm{~nm}$ with a UV detector SPD-20A (Shimadzu).

\section{Additional file}

Additional file 1. DNA seuence of primers used in this study.

\section{Abbreviations}

HMG-CoA: hydroxymethylglutaryl-coenzyme A; YM: yeast mold; PCR: polymerase chain reaction; RP-HPLC: reversed phase high-performance liquid chromatography; IPP: isopentenyl-pyrophosphate; DMAPP: dimethylallylpyrophosphate; FPP: farnesyl-pyrophosphate; GPP: geranyl-pyrophosphate; GGPP: geranylgeranyl-pyrophosphate.

\section{Authors' contributions}

KY performed the experiments and wrote the manuscript. KYH designed the study, wrote and revised the manuscript. TM and AN modified the study and assisted in performing the experiments. DS, Jl, and CO revised the manuscript. NK and AK coordinated the project. All authors read and approved the manuscript.

\footnotetext{
Author details

${ }^{1}$ Department of Chemical Science and Engineering, Graduate School of Engineering, Kobe University, 1-1 Rokkodaicho, Nada-ku, Kobe 657-8501, Japan. ${ }^{2}$ Division of Environmental and Life Sciences, Graduate Division of Nutritional and Environmental Sciences, University of Shizuoka, 52-1 Yada, Suruga-ku, Shizuoka 422-8526, Japan. ${ }^{3}$ Organization of Advanced Science and Technology, Kobe University, 1-1 Rokkodaicho, Nada-ku, Kobe 657-8501, Japan. ${ }^{4}$ Medical Device Development Laboratories, Kaneka Corporation, Takasago, Hyogo, Japan. ${ }^{5}$ Graduate School of Science, Technology, and Innovation, Kobe University, 1-1 Rokkodai-cho, Nada-ku, Kobe, Hyogo 657-8501, Japan. ${ }^{6}$ Biotechnology Development Laboratories, Kaneka Corporation, Takasago, Hyogo, Japan.
} 


\section{Acknowledgements}

We thank Dr. J. Kobayashi, Dr. T. Hasunuma, Dr. K. Sasaki, and Dr. M. Mochizuki (Graduate School of Science, Technology, and Innovation, Kobe University) for their helpful discussion. We are grateful to N. Aoki, and K. Morikawa for their technical assistance.

\section{Competing interests}

The authors declare that they have no competing interests.

\section{Availability of data and materials}

The datasets supporting the conclusions of this article are included within the article and its Additional file 1.

\section{Ethics approval and consent to participate}

This manuscript dose not report data collected from humans or animals.

\section{Funding}

This study was supported by Special Coordination Funds for Promoting Science and Technology, and the Creation of Innovation Centers for Advanced Interdisciplinary Research Areas (Innovative Bioproduction Kobe, iBioK), Ministry of Education, Culture, Sports, Science and Technology, Japan.

Received: 15 March 2016 Accepted: 7 September 2016

Published online: 13 September 2016

\section{References}

1. Vachali P, Bhosale P, Bernstein PS. Microbial carotenoids. Methods Mol Biol. 2012;898:41-59.

2. Muntendam R, Melillo E, Ryden A, Kayser O. Perspectives and limits of engineering the isoprenoid metabolism in heterologous hosts. Appl Microbiol Biotechnol. 2009:84:1003-9.

3. Westfall PJ, Pitera DJ, Lenihan JR, Eng D, Woolard FX, Regentin R, et al. Production of amorphadiene in yeast, and its conversion to dihydroartemisinic acid, precursor to the antimalarial agent artemisinin. Proc Natl Acad Sci USA. 2012;109:E111-8.

4. Ajikumar PK, Xiao WH, Tyo KE, Wang Y, Simeon F, Leonard E, et al. Isoprenoid pathway optimization for Taxol precursor overproduction in Escherichia coli. Science. 2010;330:70-4

5. Melillo E, Setroikromo R, Quax WJ, Kayser O. Production of a-cuprenene in Xanthophyllomyces dendrorhous: a step closer to a potent terpene biofactory. Microb Cell Fact. 2013;12:13.
6. Rodríguez-Sáiz M, Godio RP, Alvarez V, de la Fuente JL, Martín JF, Barredo $J$ L. The NADP-dependent glutamate dehydrogenase gene from the astaxanthin producer Xanthophyllomyces dendrorhous: use of its promoter for controlled gene expression. Mol Biotechnol. 2009;41:165-72.

7. Hara KY, Morita T, Endo Y, Mochizuki M, Araki M, Kondo A. Evaluation and screening of efficient promoters to improve astaxanthin production in Xanthophyllomyces dendrorhous. Appl Microbiol Biotechnol. 2014;98:6787-93

8. Hara KY, Morita T, Mochizuki M, Yamamoto K, Ogino C, Araki M, et al. Development of a multi-gene expression system in Xanthophyllomyces dendrorhous. Microb Cell Fact. 2014;13:175.

9. Gassel S, Schewe H, Schmidt I, Schrader J, Sandmann G. Multiple improvement of astaxanthin biosynthesis in Xanthophyllomyces dendrorhous by a combination of conventional mutagenesis and metabolic pathway engineering. Biotechnol Lett. 2013;35:565-9.

10. Castelblanco-Matiz LM, Barbachano-Torres A, Ponce-Noyola T, RamosValdivia AC, Cerda García-Rojas CM, Flores-Ortiz CM, et al. Carotenoid production and gene expression in an astaxanthin-overproducing Xanthophyllomyces dendrorhous mutant strain. Arch Microbiol. 2015:197:1129-39.

11. Mauricio N, Jennifer A, Salvador B, Dionisia S, Carla L, Marisela C, et al. Genomic organization of the structural genes controlling the astaxanthin biosynthesis pathway of Xanthophyllomyces dendrorhous. Biol Res. 2008:41:93-108.

12. Jennifer A, Salvador B, Marisela C, Carla L, Andres M, Mauricio N, et al. Cloning of the cytochrome p450 reductase (crtR) gene and its involvement in the astaxanthin biosynthesis of Xanthophyllomyces dendrorhous. BMC Microbiol. 2008;8:169.

13. Jennifer A, Ignacio R, Mauricio N, Dionisia S, Maria CR, Marcelo B, et al. Functional characterization of the Xanthophyllomyces dendrorhous farnesyl pyrophosphate synthase and geranylgeranyl pyrophosphate synthase encoding genes that are involved in the synthesis of isoprenoid precursors. PLoS ONE. 2014:9:e96626.

14. Iris L, Maria SG, Salvador B, Dionisia S, Pilar M, Marcelo B, et al. Enhancement of carotenoid production by disrupting the C22-sterol desaturase gene (CYP61) in Xanthophyllomyces dendrorhous. BMC Microbiol. 2012;12:235.

15. Gassel S, Breitenbach J, Sandmann G. Genetic engineering of the complete carotenoid pathway towards enhanced astaxanthin formation in Xanthophyllomyces dendrorhous starting from a high-yield mutant. Appl Microbiol Biotechnol. 2014;98:345-50.

16. Wery J, Gutker D, Renniers AC, Verdoes JC, van Ooyen AJ. High copy number integration into the ribosomal DNA of the yeast Phaffia rhodozyma. Gene. 1997;184:89-97.

\section{Submit your next manuscript to BioMed Central and we will help you at every step:}

- We accept pre-submission inquiries

- Our selector tool helps you to find the most relevant journal

- We provide round the clock customer support

- Convenient online submission

- Thorough peer review

- Inclusion in PubMed and all major indexing services

- Maximum visibility for your research

Submit your manuscript at www.biomedcentral.com/submit
C Biomed Central 